\title{
Influence of the variability of processing factors on the $F$-value distribution in batch retorts
}

\author{
Szabolcs Varga ${ }^{a}$, Jorge C. Oliveira ${ }^{b, *}$, Fernanda A.R. Oliveira ${ }^{c}$ \\ ${ }^{a}$ Escola Superior de Biotecnologia, Universidade Católica Portuguesa, Rua Dr António Bernardino de Almeida, 4200 Porto, Portugal \\ ${ }^{\mathrm{b}}$ Department of Food Science and Technology, University College Cork, Cork, Ireland \\ ${ }^{\mathrm{c}}$ Department of Food Engineering, University College Cork, Cork, Ireland
}

Keywords: Conduction heating; Finite elements; Monte Carlo simulation; In-pack sterilisation

\begin{abstract}
The $F$-value distribution in a batch retort processing of in-pack conduction heating foods resulting from the variability of four processing factors (initial temperature, heating time, headspace and external heat transfer coefficient) was determined by mathematical modelling. Three hundred combinations were generated from normally distributed values for each factor, following the Monte Carlo approach. The retort temperature was assumed the same for all containers and the $F$-value at the geometrical centre of containers of different dimensions was calculated by solving the governing heat transfer equations using finite elements. It was found that in the range tested the variability of the heating time and of the external heat transfer coefficient had the biggest impact on the $F$-value distribution and that the head space variability had a negligible impact. It was also found that although each influencing factor had a normal distribution, the $F$-value distribution was not normal, showing a significant tailing, being well described by a gamma function.
\end{abstract}

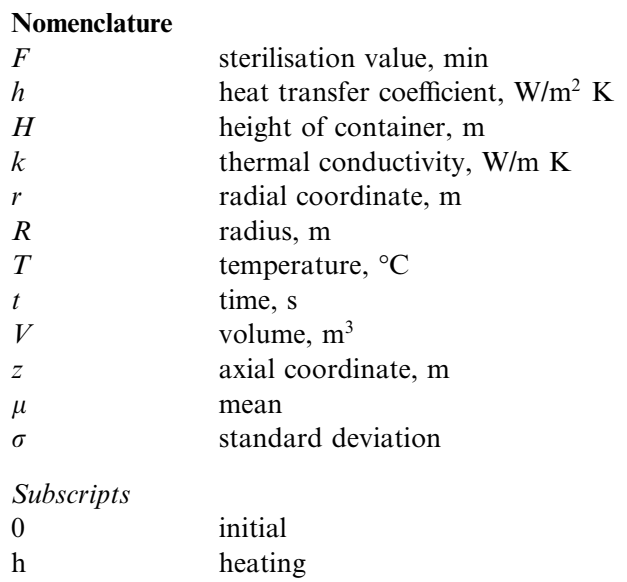

\section{Introduction}

The design and evaluation of batch retort processes are based on the application of the "cold spot" and "worst case" approach, that is, on the definition of the location in the retort where the least thermal process occurs and of the combination of other factors that would result in the lowest $F$-value in that location. However, this approach is questionable from a statistical point of view: it would be better to determine the actual process variability and $F$-value distribution and thus define critical values.

A statistically based approach requires an extensive and solid knowledge based on the impact of the processing factors on the $F$-value distribution. The retort temperature distribution is a major factor to be analysed, as required by GMP and legislation (Adams \& Hardt-English, 1990). This work is concerned only with other factors.

In literature, experimentally assessed thermal variability is mostly restricted to the measurement of the influencing factors (Hicks, 1961; Lenz \& Lund, 1977; Lund, 1978; Pflug \& Odlaug, 1978; Patino \& Heil, 1985; McGinnis, 1986; Hayakawa, Massaguer \& Trout, 1988; Lebowitz \& Bhowmik, 1989, 1990; Adams \& HardtEnglish, 1990; Park, Cables \& Collins, 1990; Tung, Britt \& Ramaswamy, 1990; Ramaswamy \& Tung, 1990), but the effect of such variability on the resulting lethality has not been often reported (Powers, Pratt, Carmon, 
Somaatadja \& Forston, 1962; Tung, Morello \& Ramaswamy, 1989; Campbell \& Ramaswamy, 1992).

Most available simulation approaches use the Monte Carlo technique to generate physical properties and thermal destruction parameters (Lenz \& Lund, 1977b; Hayakawa et al., 1988; Xie \& Sheard, 1995). With a few exceptions, $F$-value distribution due to the variability in processing conditions such as headspace has not been studied. There are other statistical approaches available to evaluate thermal process variability that have been reported (Wang, Wolfe \& Hayakawa, 1991; Hayakawa, Jianjun \& de Massaguer, 1996; Nicolai \& Baerdemaeker, 1992a,b, 1993, 1996; Nicolai, Schellekens, Martens \& de Baerdemaeker, 1994; Johns, 1992). The major disadvantage of these methods is that they are not capable to assess the entire $F$-value distribution so that its type cannot be evaluated.

A very important question in this framework is the number of experiments or mathematical simulations required to achieve a reliable result. In most published works a sample size of about 100 was used. Hayakawa et al. (1988) used a sample size of 60 , but the result correlated neither to the normal nor to the gamma distribution accurately. Breman (1973) recommended that a sample size of 100 or more is required to distinguish between two very different distributions such as uniform and normal distributions. A large amount of data is therefore required. A better understanding of the heat distribution in an industrial scale retort, and in particular the knowledge of how and to what extent the relevant factors affect the $F$-value variability, would provide the possibility to reduce the amount of experimental effort necessary to evaluate a thermal process.

In this work a fully modelling approach is followed to analyse the influence of process variability on the $F$-value distribution due to four processing factors as simple random variables (initial temperature $\left(T_{0}\right)$, external heat transfer coefficient $(h)$, headspace volume $\left(V_{\text {headsp }}\right)$ and heating time $\left.\left(t_{\mathrm{h}}\right)\right)$. The necessary number of sample size for different types of distributions is also analysed.

\section{Materials and methods}

\subsection{Finite element heat transfer model}

A numerical finite element (FE) model was used to predict the temperature distribution in a conduction heating cylindrical shaped body. Detailed mathematical description of the method is given by Segerlind (1984) and in software manuals (ANSYS Manual, ANSYS, Houston, USA). In this work, the conduction heating problem was solved using Fourier's second law for the appropriate geometry (finite cylinder) with the following boundary conditions:
From Fourier's law:

$\alpha\left(\frac{\partial^{2} T}{\partial z^{2}}+\frac{1}{r} \frac{\partial^{2}(r T)}{\partial r^{2}}\right)=\frac{\partial T}{\partial t}$.

Limit conditions:

- initial conditions: $t=0, T=T_{0} \quad \forall x, r$,

- symmetry condition: $r=0, \frac{\partial T}{\partial r}=0 \quad \forall t$,

- boundary conditions:

(a) $x=0 ; H, \quad-\left.k \frac{\partial T}{\partial x}\right|_{x=0, H}=h\left(T-T(t)_{R}\right)$,

(b) $r=R, \quad-\left.k \frac{\partial T}{\partial r}\right|_{r=R}=h\left(T-T(t)_{R}\right)$.

$T(t)_{\mathrm{R}}$ represents the retort temperature which was taken as a step function of time. A constant value of $130^{\circ} \mathrm{C}$ and $20^{\circ} \mathrm{C}$ were selected for heating and cooling, respectively.

Given the symmetry of the problem, only one-quarter of a can was modelled, dividing it in 112, 4-node quadrilateral elements, with decreasing size towards the boundary. The finite element problem was solved using the ANSYS5.3 (Houston, USA) commercial software package. Four standard container sizes were considered: $211 \times 304,211 \times 109,307 \times 113$ and $307 \times 512$ (American standard notation). The accuracy of the model was checked by comparing its results with those of analytical solutions of Fourier's 2nd law at limit conditions. The physical properties considered were typical of food products, and correspond to an $8 \%$ bentonite solution, commonly used as a conduction heating food simulant (density $1052 \mathrm{~kg} / \mathrm{m}^{3}$, heat conductivity $0.768 \mathrm{~W} / \mathrm{m} \mathrm{K}$, specific heat $3810 \mathrm{~J} / \mathrm{kg} \mathrm{K}$ ). For the headspace, the physical properties of air were used. The simulated processing time was $80 \mathrm{~min}$. This was sufficient for the center temperature of the can to reach $125-128^{\circ} \mathrm{C}$. The process variability was evaluated by the $F$-value distribution calculated for the geometric centre of the container, using the general method, for a target microorganism with a $z$-value of $10^{\circ} \mathrm{C}$.

\subsection{Random variables}

The Monte Carlo method has been widely assigned to methods that apply models with random or random like outcome, mostly simple random variates from a previously defined statistical distribution (Ripley, 1987). A computer program was written in FORTRAN 77 to simulate random sampling from different distributions, using the composition technique (Law \& Kelton, 1991).

Four processing factors were considered: $T_{0}, h, V_{\text {headsp }}$ and $t_{\mathrm{h}}$. The mean and standard deviation for the initial temperature $\left(\mu_{T_{0}}=40^{\circ} \mathrm{C}, \sigma_{T_{0}}=2.2^{\circ} \mathrm{C}\right)$ were taken from 
literature data (Hayakawa et al., 1988). The heating time $\left(\mu_{t_{\mathrm{b}}}=55 \mathrm{~min}, \sigma_{t_{\mathrm{h}}}=2 \mathrm{~min}\right)$ and the headspace volume $\left(\mu_{V}=10 \%, \sigma_{v}=2.5 \%\right)$ were arbitrarily selected. The heat transfer coefficient $\left(\mu_{\mathrm{h}}=164 \mathrm{~W} / \mathrm{m}^{2} \mathrm{~K}\right.$, $\sigma_{\mathrm{h}}=28.2 \mathrm{~W} / \mathrm{m}^{2} \mathrm{~K}$ ) was based on experimental measurements on a pilot scale retort, which verified a close to normal distribution (Varga, 1998). It was assumed that each factor was independent of all the others and normally distributed, since there are no experimental data suggesting otherwise. It is noted that Hayakawa et al. (1988) recommends the use of the gamma distribution instead of the normal in order to avoid possible negative values for the generated variability. However, in this case, according to the mean and standard deviation values considered, it is very unlikely to obtain observations lower than zero.

\section{Results and discussion}

\subsection{Random variety generation}

The computer program written for the generation of random samples from different distributions was tested using the statistical program package STATGRAPHYCS 5.0 (Rockville, USA) and the minimum number of samples required by each distribution in order to obtain reliable results was determined. Random numbers were generated from each distribution with sample sizes of 10, 50, 100, 500, 1000 and 2000, and then a distribution function was fitted to the results. Chisquared tests were carried out to assess whether the results of these fits were significantly different from the theoretical distributions that originated the data, at a $95 \%$ confidence level.

The results showed that all the distributions were simulated very accurately with more than 100 data, except the gamma distribution in which case the fitting was not accurate even for very large sample sizes. The random number generator of STATGRAPHYCS showed better results for gamma varieties but was also quite inaccurate even for a sample size of 1000 . This also implies that the recommendation by Hayakawa et al. (1988) of using the gamma distribution to describe the variability of the influencing factors would require a very large sample size in order to represent the target distribution with acceptable precision. Table 1 shows the sample sizes that were deemed adequate for the different distributions (statistical similarity of all distribution parameters at $95 \%$ confidence level). It is noted that ANSYS also has random number generation capabilities for some continuous distributions such as normal and uniform. Its performance was compared to the program developed using the Kolmogorov-Smirnov test and the STATGRAPHYCS nonparametric method for randomness, and it was found that the latter approxi- mated slightly better for both the uniform and normal distributions than the ANSYS generator.

\subsection{Thermal process simulation}

A sample size of 300 normally distributed random values was generated for each factor. The mean and standard deviations of the simulated were very close to the targets, as seen in Table 1.

The effect of each variable on the $F$-value value was first evaluated for each can size using a $2^{4}$ full factorial design. The low and high values of the parameters were selected at $\mu \pm 1 \sigma$. Standardised Pareto charts constructed from the results are shown in Figs. 1-4. The vertical line corresponds to the $95 \%$ confidence limit. The effects that exceed this limit are significant. It is noted that the third and higher order interactions were included in the random error calculation (white noise). It is clear from the figures that all the individual effects are far beyond the significance limit, except the headspace volume for "tall" can sizes $(211 \times 304$ and

Table 1

Sample sizes, in round figures, of the different distributions required to obtain good agreement between the input and the simulated distributions after fitting the random data back to the original distribution function

\begin{tabular}{ll}
\hline Distribution & Sample size \\
\hline Normal & 50 \\
Lognormal & $50-100$ \\
Weibull & 100 \\
Gamma & 1000 \\
Exponential & 100 \\
Beta & $100-500$ \\
\hline
\end{tabular}

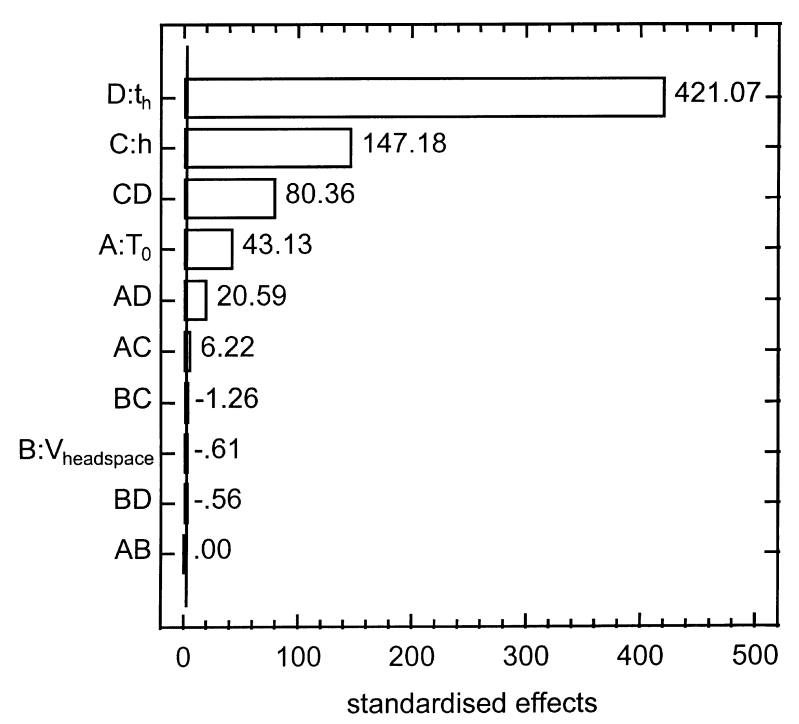

Fig. 1. Standardised Pareto chart for can size $211 \times 304$ (American standard). 


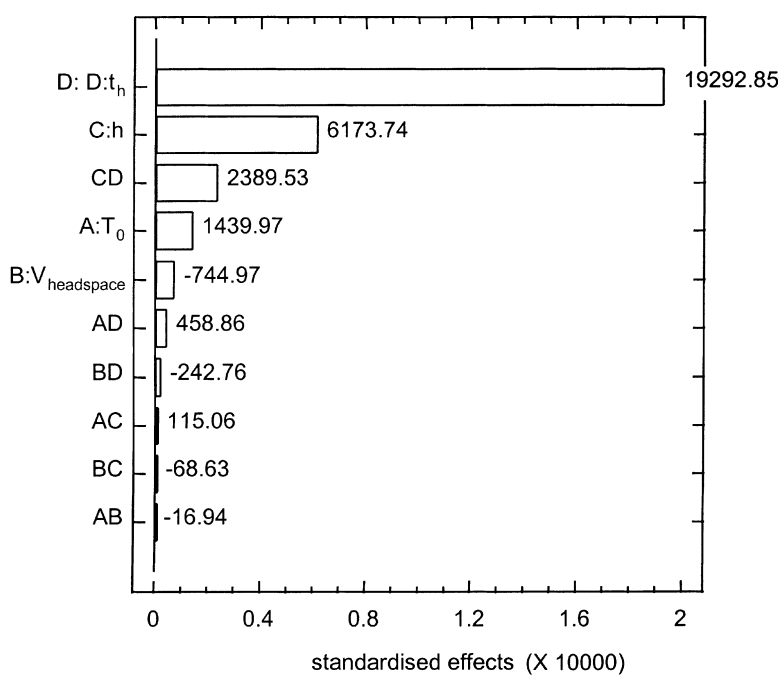

Fig. 2. Standardised Pareto chart for can size $211 \times 109$ (American standard).

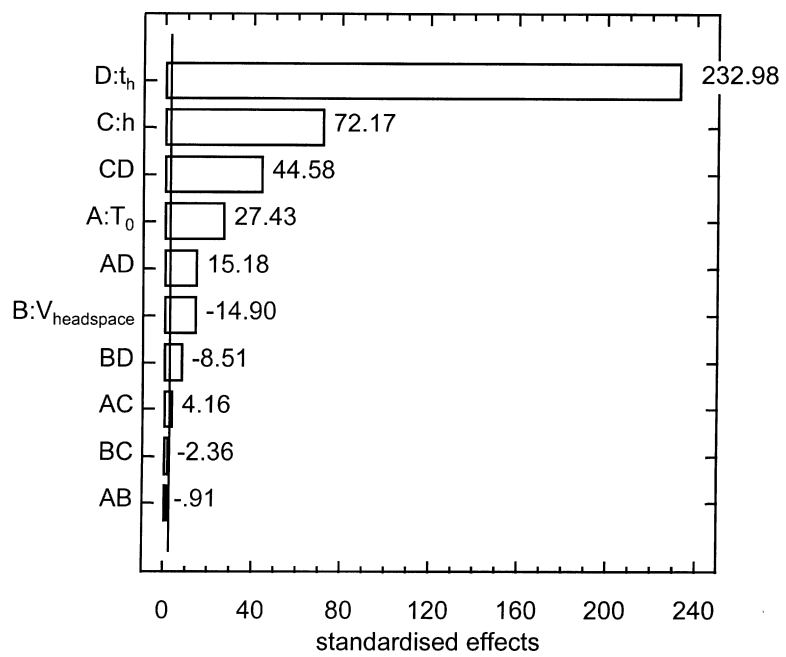

Fig. 3. Standardised Pareto chart for can size $307 \times 113$ (American standard).

$311 \times 512$ ). This is probably due to the fact that the higher order interactions that were used to calculate the random error were very small, but Figs. 1-4 can still be used to assess the relative importance of the factors and their second order interactions. The most important factors within the range of variability considered were the heating time and the heat transfer coefficient, showing also a relevant interaction. The effect of the initial temperature was also significant for all can types. The headspace volume was found to be the least important factor influencing the lethality value. Its effect was more relevant for the "short" can types $(211 \times 109$ and $307 \times 113$ ) than for "tall" containers. However, it is noteworthy that, although the headspace volume did not have a very important effect, it might change the

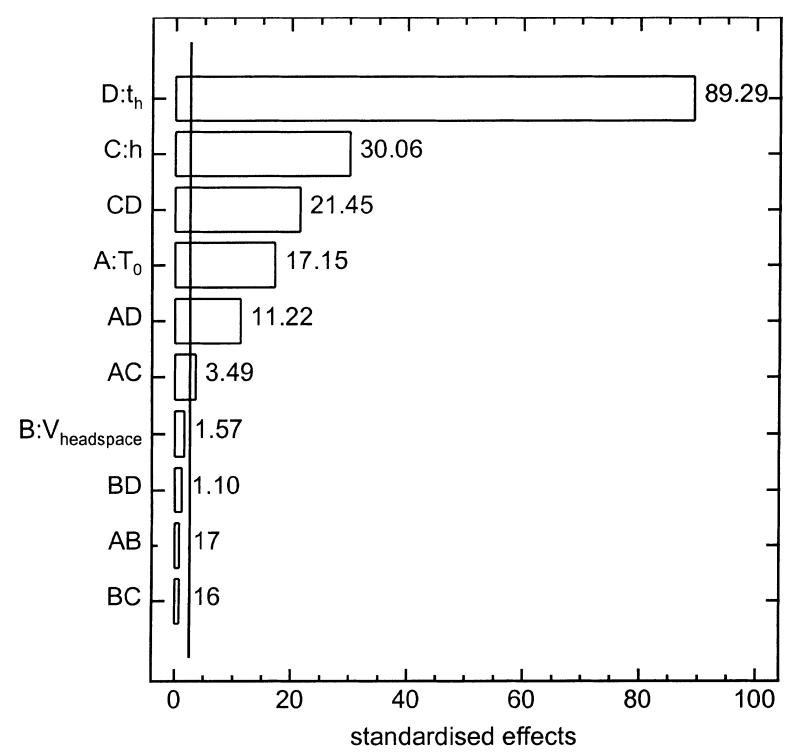

Fig. 4. Standardised Pareto chart for can size $307 \times 512$ (American standard)

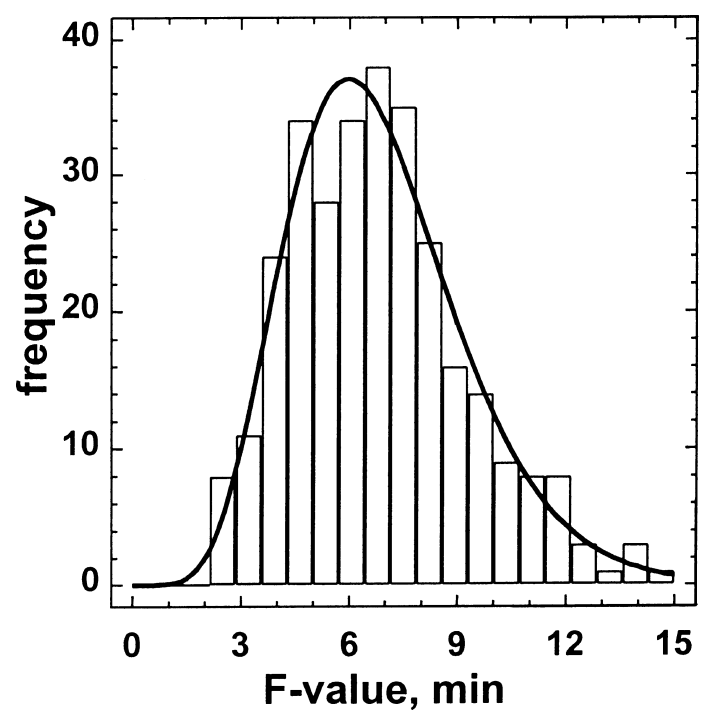

Fig. 5. $F$-values in the geometric centre of a $211 \times 304$ (American standard) can and the corresponding input factor distributions. The solid line is the fitted gamma ( $F$-value) distribution.

exact location of the least lethality point inside the package.

Only one "tall" $(211 \times 304)$ and one "short" $(307 \times 113)$ can size were selected to determine the $F$-value distribution, since similar can heights showed similar results in the factorial design analysis. The calculated $F$-value distribution is shown in Fig. 5 for the $211 \times 304$ can. It can be seen that although all input variables were normally distributed, the resulting $F$-value distribution was clearly tailed to the right. In fact, it was found that the gamma distribution fitted the $F$-value distribution better than normal or Weibull 


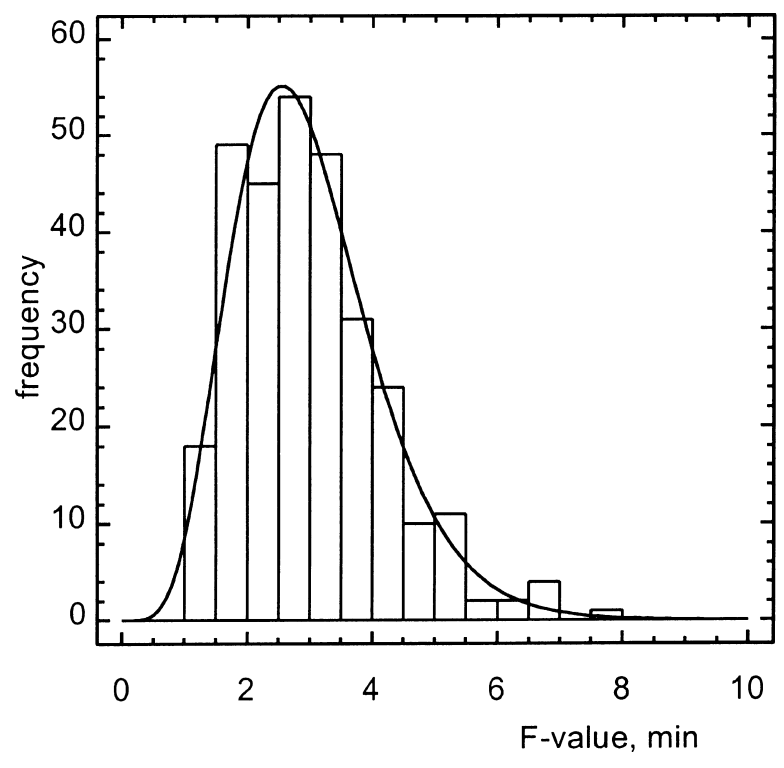

Fig. 6. Simulated lethality distribution in a $307 \times 113$ can (American standard). The solid line is the fitted gamma distribution. Input value distributions are the same as in Fig. 5.

function. The latter two did not result in a satisfactory fit according to both a chi-squared and a KolmogorovSmirnov test. Similar results were obtained by Hayakawa et al. (1988), but with a more pronounced tailing. The $F$-value distribution for the $307 \times 113$ can is represented in Fig. 6. It is clear that the same input distribution resulted in an $F$-value histogram of similar shape for the "short" can. It was also found that the simulated $F$-value distribution also skewed to the right, and the gamma distribution described the behaviour of the $F$-value distribution significantly better, while the normal and Weibull functions were not acceptable, according to the tests mentioned above.

The summary statistics of both $F$-value distributions are given in Table 2. The average $F$-value in the larger can was roughly two times lower, but the standard deviation and the coefficient of variation $(\mathrm{CV})$ remained approximately the same. The relative range ((max-min)/ average) is a measure of the relative extreme dispersion

Table 2

Summary statistics of the simulated lethality distributions $\mathrm{s}^{\mathrm{a}}$

\begin{tabular}{lll}
\hline Can size & $211 \times 304$ & $307 \times 113$ \\
\hline Average (min) & 6.85 & 2.99 \\
Standard deviation (min) & 2.44 & 1.18 \\
Minimum (min) & 2.46 & 1.02 \\
Maximum (min) & 14.38 & 7.82 \\
Relative range (\%) & 174 & 227 \\
$95 \%$ confidence limit (min) & $2.93-12.40$ & $1.18-5.65$ \\
CV (\%) & 35.66 & 39.39
\end{tabular}

${ }^{a}$ Retort programme: instantaneous come-up, constant holding temperature $130^{\circ} \mathrm{C}$, instantaneous come-down, constant cooling temperature $20^{\circ} \mathrm{C}$, process time $80 \mathrm{~min}$. of the data, and was considerably smaller for the "tall" can shape. In Table 2, the confidence interval was calculated based on the fitted gamma distribution, and the values correspond to the $F$-values where the area defined by the density curve is $2.5 \%$ and $97.5 \%$ of the total area, that is, $95 \%$ of the observations are contained between these two values.

Further data analysis was carried out to identify the importance of the input variables on the $F$-value distribution. The component effects, for both can sizes, are depicted in Figs. 7 and 8. This is a so-called componentplus-residual plots obtained by multiple regression and plotting the residuals around a line defined by multiplying the estimated component effect by the centred value of the independent variables, in this case the influencing factors (Breman, 1973). The results are in agreement with the factorial design, however also provide information about the spread of the lethality value around each effect. It can be seen in Fig. 7 that the heat transfer coefficient and the heating time had the strongest contribution to the $F$-value variability, as these factors resulted in the smallest dispersion around the estimated component effect. It is also obvious that this dispersion was not random, since it has a curvilinear shape due to the effect of the other factors. The effect of the initial temperature was less relevant, but there is an obvious tendency for an increase with $T_{0}$. It is clear from the figure that the headspace did not practically affect the lethality value. Fig. 8 indicates very similar patterns, although the component effects are slightly less emphasised.

These results could be expected from the importance of the different factors on the temperature evolution in the centre of the can. A longer process time will imply that a higher temperature is reached in the centre of the can and/or that it will be exposed to the higher (holding) temperature for a longer time (with obvious impact on a higher $F$-value); the external heat transfer coefficient can have a significant effect when it is low enough to impair the heating; a higher initial temperature will imply that the holding temperature is reached sooner in the centre of the can (shorter come-up time), which will therefore be subjected to the highest temperature for a longer period of time (as the total processing time is the same), and therefore a higher $F$-value will result; the head peace has a small influence. The results therefore imply that the greater the importance of a factor on the temperature evolution in the container, the greater the importance of its variability, which is a reasonable conclusion.

\section{Conclusions}

Within the range of variability considered, the heating time and the heat transfer coefficient were the two most critical factors, while the headspace volume variability affected the sterilisation value the least. 

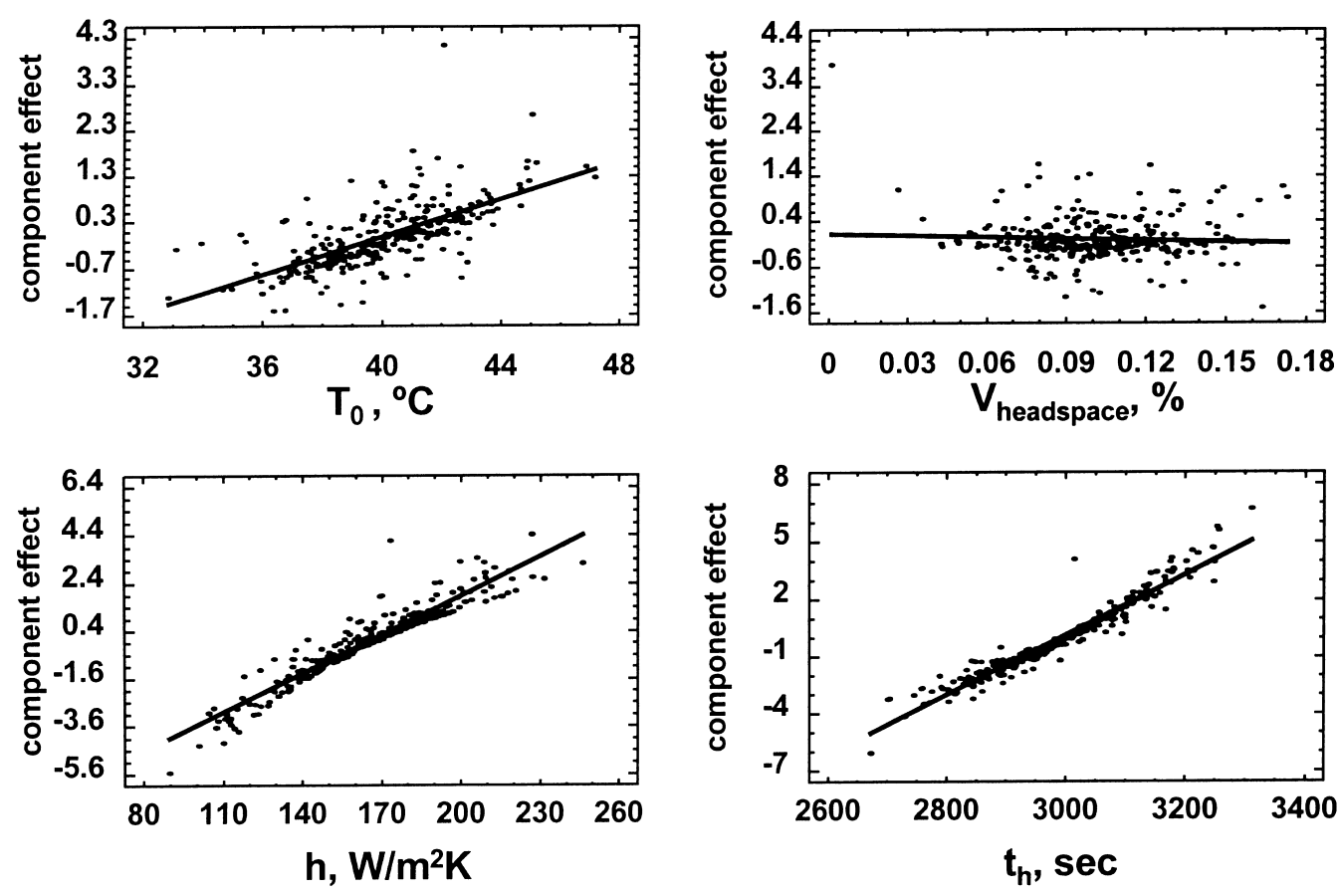

Fig. 7. Component effects for the $211 \times 304$ can.
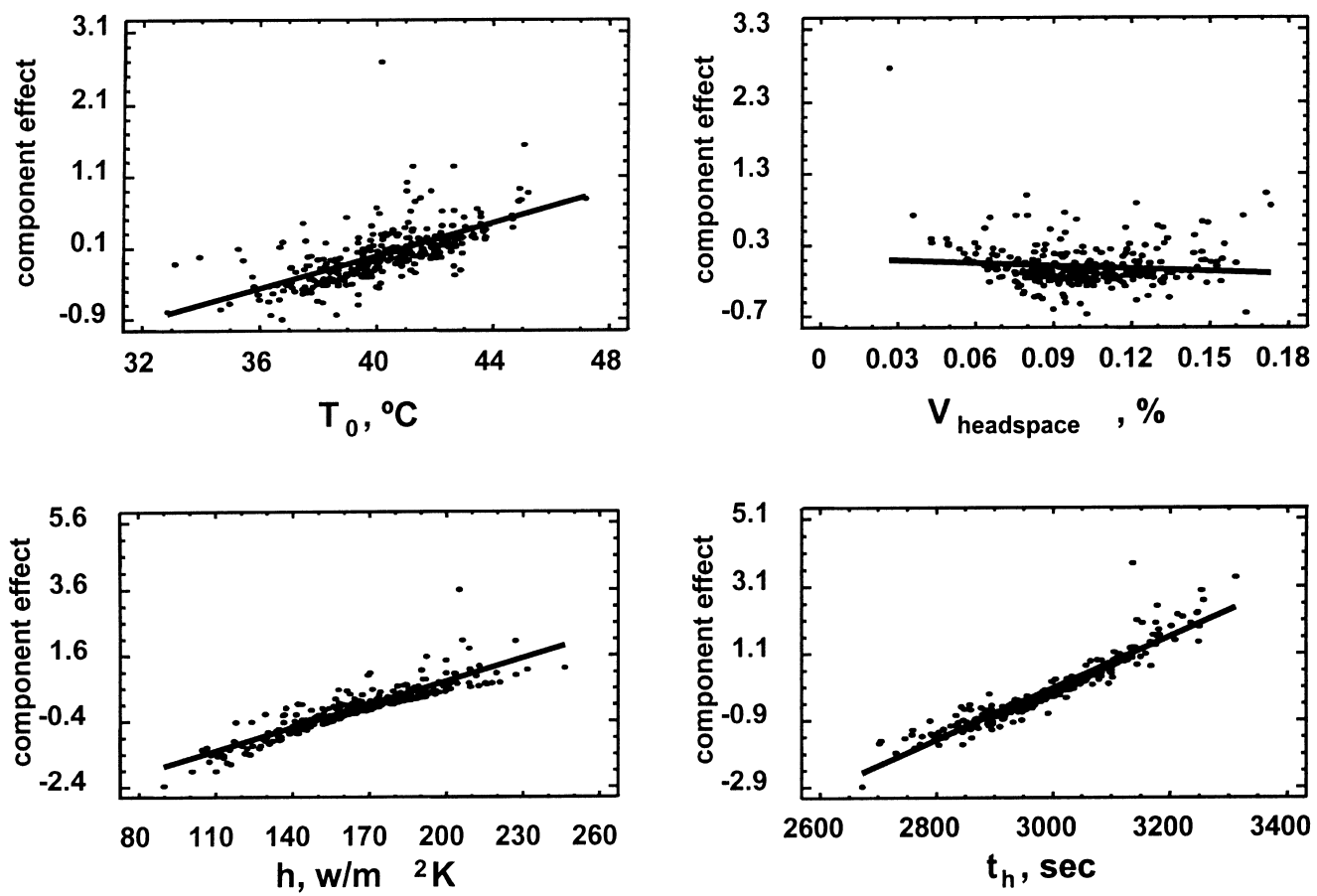

Fig. 8. Component effects for the $307 \times 113$ can.

The $F$-value distribution was clearly not normal, but tailed, and could be approximated by the gamma distribution. The tailing was more relevant in the larger size. The coefficients of variation were high $(35.66 \%$ and $39.39 \%$ ).

\section{Acknowledgements}

The authors would like to acknowledge the European Commission, AIR programme, for financial support, through project AIR2-CT94-1017. The first author 
would also like to acknowledge financial support from Fundação para Ciência e Tecnologia within the framework of the PRAXIS XXI program.

\section{References}

Adams, H. W., \& Hardt-English, P. K. (1990). Determining temperature distribution in cascading water retorts. Food Technology, 12, $110-112$.

Breman, L. (1973). Statistics: with a view toward application. Boston: Houghton Miflin.

Campbell, S., \& Ramaswamy, H. S. (1992). Distribution of heat transfer rate and lethality in a single basket water cascade retort. Journal of Food Process Engineering, 15, 31-48.

Hayakawa, K. I., Massaguer, P., \& Trout, R. J. (1988). Statistical variability of thermal process lethality in conduction heating food computerized simulation. Journal of Food Science, 53, $1887-1893$.

Hayakawa, K., Jianjun, Wang, \& de Massaguer, P. R. (1996). Simplified predictive equations for variability of thermal process lethality. Journal of Food Process Engineering, 19, 289-300.

Hicks, E. W. (1961). Uncertainties in canning process calculations. Journal of Food Science, 26, 218-226.

Johns, W. R. (1992). Simulation of food processes with uncertain data. Paper No. 1. Presented at Food Engineering in a Computer Climate, Cambridge, UK, March 30-April 1.

Law, A. M., \& Kelton, W. D. (1991). Simulation, modelling and analysis. New York: McGraw-Hill.

Lebowitz, S. F., \& Bhowmik, S. R. (1990). Effect on retortable pouch heat transfer coefficients of different thermal processing stages and pouch material. Journal of Food Science, 55, 1421-1424.

Lebowitz, S. F., \& Bhowmik, S. R. (1989). Determination of retortable pouch heat transfer coefficients by optimization method. Journal of Food Science, 54, 1407-1412.

Lenz, M. K., \& Lund, D. B. (1977). The lethality-Fourier number method: confidence intervals for calculated lethality and massaverage retention of conduction-heating canned foods. Journal of Food Science, 42, 1002-1007.

Lund, D. B. (1978). Statistical analysis of thermal process calculations. Food Technology, 32, 76-78.

McGinnis, D. S. (1986). Surface heat transfer distribution in a weir type pressurized water retort for processing foods in flexible retort pouches. Canadian Institute of Food Science and Technology Journal, 19, 45-52.

Nicolai, B. M., \& de Baerdemaeker, J. (1996). Sensitivity analysis with respect to the surface heat transfer coefficient as applied to thermal process calculations. Journal of Food Engineering, 28, 21-33.

Nicolai, B. M., \& de Baerdemaeker, J. (1993). Computation of heat conduction in materials with random variable thermophysical properties. International Journal for Numerical Methods in Engineering, 36, 523-536.

Nicolai, B. M., \& de Baerdemaeker, J. (1992a). Simulation of heat transfer in foods with stochastic initial and boundary conditions. Food and Bioproducts Processing, 70, 78-82.

Nicolai, B. M., \& de Baerdemaeker, J. (1992b). Stochastic finite element analysis of conduction heat transfer in foods with random field thermophysical properties. In F. Maceri, \& G. Iazeolla, Eurosim '92 Reprints (pp. 539-544). presented at Eurosim '92, Capri, Italy, September 29-October 2.

Nicolai, B. M., Schellekens, M, Martens, T., \& de Baerdemaeker, J. (1994). Computer-aided design of cook-chill foods under uncertain conditions. In R. P. Singh, \& F. A. R. Oliverira. Minimal Processing of Foods and Process Optimization: an Interface (pp. 293-312). Boca Raton: CRC.

Park, D. J., Cables, L. J., \& Collins, K. M. (1990). Determining temperature distribution in rotary, full-immersion, hot-water sterilizers. Food Technology, 44, 113-118.

Patino, H., \& Heil, J. R (1985). A statistical approach to error analysis in thermal process calculations. Journal of Food Science, 50, 1110 1114.

Pflug, I. J., \& Odlaug, T. E. (1978). A review of $z$ and $F$ values used to ensure the safety of low-acid canned food. Food Technology, 32, $63-70$.

Powers, J. J., Pratt, D. E., Carmon, J. L., Somaatadja, D., \& Forston, F. C. (1962). Application of extreme-value methods and other statistical procedures to heat-penetration data. Food Technology, 16, 80-89.

Ramaswamy, H. S., \& Tung, M. A. 1990. Heat transfer coefficients for water immersion heating media in vertical retorts. In W. E. L. Spiess, \& H. Schubert, Engineering and Food, vol. 2 (pp. 85-94). London: Elsevier.

Ripley, B. D. (1987). Stochastic Simulation. New York: Wiley.

Segerlind, L. J. (1984). Applied finite element analysis. New York: Wiley.

Tung, M. A., Britt, I. J., \& Ramaswamy, H. S. (1990). Food sterilization in steam/air retorts. Food Technology, 44, 105-109.

Tung, M. A., Morello, G. F., \& Ramaswamy, H. S. (1989). Food properties, heat transfer conditions and sterilization considerations in retort processes. In R. P. Singh, \& A. G. Medina, Food properties and computer-aided engineering of food processing systems (pp. 4971). Dordrecht: Kluwer.

Varga, Sz. (1998) A new approach to model and assess process variability in batch retorts. Ph.D. thesis. Escola Superior de Biotecnologia, Porto, Portugal.

Wang, J., Wolfe, R. R., \& Hayakawa, K. (1991). Thermal process lethality variability in conduction-heated foods. Journal of Food Science, 56, 1424-1428.

Xie, G., \& Sheard, M. A. (1995). Estimation of confidence interval of pasteurizing values of conduction-heated sous vide food in a combination oven. International Journal of Food Science and Technology, 30, 745-755. 